\title{
Development and Sensory Evaluation of Beta Carotene Rich Food Preparations Using Underexploited Carrot Greens
}

\author{
Tarvinder Jeet Kaur* and G.K.Kochar** \\ Department of Home Science, Kurukshetra University, Kurukshetra 136 119, Haryana, India \\ E-mail:*<tarvinkuk7@yahoo.com>, **<kochargk@yahoo.com>
}

KEYWORDS Best Acceptable. Incorporation. Organoleptically. Overall Acceptability Scores.

\begin{abstract}
Carrot greens are the most under-exploited class of greens despite of high nutritional value. In daily diet an increased intake of these inexpensive greens can be one of the strategies for improving the nutritional status. Keeping this in view, the present study was undertaken with the objective to develop commonly consumed food preparations by separately incorporating fresh and dry carrot greens, applying different cooking methods. Levels of incorporation of fresh carrot greens in different food preparations ranged 40 to 80 per cent whereas powder of dry carrot greens was added in different preparations at 7, 8 and 9 per cent levels. Organoleptic evaluation of all food preparations were conducted by a panel of ten judges using Hopkin's seven point scale. In fresh carrot greens incorporated food preparations, the maximum and most acceptable level $(80 \%)$ of incorporation of under exploited carrot greens was in saag and minimum (40\%) in puri and kadhi. In dry carrot greens incorporated food preparations, the most and best level (9\%) of incorporation of greens was in mathri and minimum (7\%) in matrey. Among all the fresh carrot greens incorporated food preparations, gulabjamun scored highest $(6.96 \pm 0.27)$ overall acceptability scores while laddoo scored minimum $(5.52 \pm 0.68)$. In dry carrot greens incorporated food preparations tacques scored highest $(6.31 \pm 0.28)$ and laddoo scored minimum $(5.98 \pm 0.69)$ overall acceptability scores. Beta carotene content in all food preparations increased significantly $(\mathrm{P}<0.01)$ with incorporation of fresh as well as dry carrot greens.
\end{abstract}

\section{INTRODUCTION}

Available sub clinical data indicate the high prevalence of Vitamin A deficiency among children (NPCB 1981; Thylefors 1985; UNDP 1992). UNICEF and WHO consider that mortality may reduce by 23 per cent on average by improving the vitamin A status of young children with marginal deficiency. Dietary approaches are needed to replace supplementation programs, ensuring sustainability and adequate coverage. Therefore it is essential that locally available materials which are inexpensive but highly nutritious be used as a vehicle to improve the nutritional status (Negi and Roy 2004).

Carrot, commonly consumed root vegetable is accessible throughout the year in India and other parts of the world. Green leaves of carrot are generally discarded while these leaves are rich source of $\beta$ carotene as well as iron, calcium, ascorbic acid, folic acid and in appreciable amount of other minerals (Ramadasmurthy and Mohanram 1984; ICMR 1989; Gopalan et al. 2004). In daily diet, an increased intake of these inexpensive under exploited greens can be one of the strategies for improving the nutritional status (Gopalan1992a,1996b).

Recognizing the need for the identification of such unconventional sources which are nutritious, inexpensive, locally available and may be helpful in achieving nutritional security, the present study was undertaken with the objective to develop commonly consumed food preparations by incorporating $\beta$ carotene rich, inexpensive under exploited greens of carrot, using different methods (deep fat frying, shallow fat frying, pressure cooking, boiling, steaming and roasting) of cooking.

\section{MATERIALS AND METHODS}

Materials: The carrot greens were procured from the kitchen garden of Kurukshetra (Haryana), India. They were obtained during the winter season (January-February).

Processing of the Sample: The edible portions of the greens of carrot were separated from stalks, cleaned, washed under running tap water followed by distilled water and dried between folds of filter paper. The fresh carrot greens were divided into two batches and processed as follows:

- cut into even sized pieces with stainless knife and used for different preparations.

- dried in hot air oven at $50^{\circ} \mathrm{C}$ for $16-18$ hours, powdered and stored in airtight containers under refrigerated temperature for further use. The dried sample as well as fresh sample were 
subsequently incorporated into different commonly consumed food preparations.

Development of Food Preparations: Commonly consumed food preparations were prepared by the procedure of Kaur (2007) using different cooking methods commonly applied in homes by separately incorporating fresh and dry carrot greens. Levels of incorporation of fresh greens of carrot in different food preparations were 40,50, 60, 70 and 80 per cent, whereas, powder of dry carrot greens was added in different recipes at 7,8 and 9 per cent levels.

Sensory Evaluation: All the developed food preparations were presented to a panel of ten judges and were evaluated organoleptically using Hopkin's seven point scale (1950).

$\beta$ Carotene Content: $\beta$ carotene content in controlled as well as carrot greens incorporated food preparations was calculated from the values per $100 \mathrm{~g}$ of edible portions by using nutritive value of Indian foods (Gopalan et al. 2004).

Statistical Analysis: The data obtained was analyzed statistically (Gupta and Saini 1987). Mean and standard error of mean were calculated for each studied variable. The interpretation of the data so obtained was done by analysis of variance (ANOVA) test. Level of significance was accepted at $\mathrm{P} \leq 0.01$.

\section{RESULTS AND DISCUSSION}

Sensory Evaluation: The sensory evaluation of the food preparations made by the incorporation of greens revealed that all the food products developed were organoleptically acceptable. However, the acceptable level of incorporation of greens varied in different food preparations. It has also been noticed that when the level of incorporation of greens increased beyond the accepted levels in any preparations, the mean scores for the organoleptic evaluation for appearance, color, texture, taste, flavor and overall acceptability decreased.

Fresh Carrot Greens Incorporated Food Preparations: In fresh greens incorporated food preparations, i.e., puri and kadhi, the most acceptable level of incorporation was 40 per cent and the scores for overall acceptability awarded by the panel of judges were $6.20 \pm 0.52$ and $6.80 \pm$ 0.21 , respectively. For idli, gulabjammun, pancake and rabri, the most acceptable level of incorporation was 50 per cent of fresh carrot greens and the respective scores for overall acceptability ranged from $6.20 \pm 0.41$ (pancake) to $6.96 \pm 0.27$ (gulabjamun). At 60 per cent level of incorporation of fresh greens, chutney, cutlets, chilla, pulao and gulgulae were best acceptable

Table 1: Food preparations prepared with different methods of cooking by incorporating fresh and dry leaves powder of Underexploited Carrot Greens (UCG)

\begin{tabular}{|c|c|c|c|c|c|}
\hline \multirow[t]{2}{*}{ Food preparations } & \multicolumn{2}{|c|}{ Nature of preparations } & \multicolumn{2}{|c|}{ Leaves incorporated } & \multirow[t]{2}{*}{ Method of processing } \\
\hline & Salty & Sweet & Fresh & Dry & \\
\hline Puri & Salty & - & Fresh & - & \\
\hline Cutlets & Salty & - & Fresh & - & \\
\hline Mathri & Salty & - & Fresh & - & \\
\hline Matrey & Salty & - & Fresh & - & \\
\hline Tacques & Salty & - & Fresh & - & \\
\hline Titbits & Salty & - & Fresh & - & \\
\hline Gulabjamun & - & Sweet & Fresh & - & Deep fat frying \\
\hline Gulgulae & - & Sweet & Fresh & - & \\
\hline Mathri & Salty & - & - & Dry & \\
\hline Matrey & Salty & - & - & Dry & \\
\hline Tacques & Salty & - & - & Dry & \\
\hline Titbits & Salty & - & - & Dry & \\
\hline Chilla & Salty & & Fresh & - & \\
\hline Pancake & - & Sweet & Fresh & - & Shallow fat frying \\
\hline Soup & Salty & - & Fresh & - & \\
\hline Saag & Salty & - & Fresh & - & \\
\hline Pulao & Salty & - & Fresh & - & Pressure cooking \\
\hline Sweet Pulao & - & Sweet & Fresh & - & \\
\hline Kadhi & Salty & - & Fresh & - & \\
\hline Rabri & - & Sweet & Fresh & - & Boiling \\
\hline Idli & Salty & - & Fresh & - & Steaming \\
\hline Laddoo & - & Sweet & Fresh & - & Blanching+Roasting \\
\hline Laddoo & - & Sweet & - & Dry & Roasting \\
\hline
\end{tabular}


Table 2: Sensory evaluation of different food preparations with incorporation of fresh underexploited greens of carrot

\begin{tabular}{|c|c|c|c|c|c|c|c|}
\hline $\begin{array}{l}\text { Food } \\
\text { prepara- } \\
\text { tions }\end{array}$ & $\begin{array}{l}\% \text { level } \\
\text { of incor- } \\
\text { poration }\end{array}$ & Appearance & Color & Texture & Taste & Flavour & $\begin{array}{c}\text { Overall } \\
\text { acceptability }\end{array}$ \\
\hline Chutney & 50 & $6.10 \pm 0.23$ & $6.20 \pm 0.35$ & $6.10 \pm 0.30$ & $6.40 \pm 0.42$ & $6.50 \pm 0.24$ & $6.30 \pm 0.38$ \\
\hline \multirow[t]{2}{*}{ (Sa) } & 60 & $6.20 \pm 0.28$ & $6.40 \pm 0.26$ & $6.30 \pm 0.36$ & $6.60 \pm 0.38$ & $6.70 \pm 0.35$ & $6.40 \pm 0.44$ \\
\hline & 70 & $6.05 \pm 0.19$ & $6.10 \pm 0.29$ & $6.10 \pm 0.43$ & $6.10 \pm 0.39$ & $6.50 \pm 0.20$ & $6.20 \pm 0.58$ \\
\hline Puri & 30 & $6.30 \pm 0.51$ & $6.30 \pm 0.65$ & $5.90 \pm 0.45$ & $6.10 \pm 0.19$ & $5.90 \pm 0.56$ & $5.90 \pm 0.41$ \\
\hline \multirow[t]{2}{*}{$(\mathrm{Sa})$} & 40 & $6.50 \pm 0.48$ & $6.40 \pm 0.52$ & $6.00 \pm 0.32$ & $6.20 \pm 0.23$ & $6.00 \pm 0.64$ & $6.20 \pm 0.52$ \\
\hline & 50 & $6.30 \pm 0.42$ & $6.20 \pm 0.61$ & $5.90 \pm 0.38$ & $6.00 \pm 0.25$ & $6.00 \pm 0.59$ & $6.10 \pm 0.64$ \\
\hline Cutlets & 50 & $6.00 \pm 0.56$ & $6.20 \pm 0.35$ & $6.10 \pm 0.56$ & $6.10 \pm 0.23$ & $5.90 \pm 0.58$ & $6.10 \pm 0.46$ \\
\hline \multirow[t]{2}{*}{$(\mathrm{Sa})$} & 60 & $6.20 \pm 0.45$ & $6.40 \pm 0.28$ & $6.20 \pm 0.52$ & $6.30 \pm 0.35$ & $6.10 \pm 0.49$ & $6.20 \pm 0.63$ \\
\hline & 70 & $5.89 \pm 0.42$ & $6.10 \pm 0.33$ & $5.90 \pm 0.43$ & $6.00 \pm 0.30$ & $5.80 \pm 0.46$ & $5.90 \pm 0.52$ \\
\hline Chilla & 50 & $6.30 \pm 0.31$ & $6.20 \pm 0.56$ & $5.90 \pm 0.42$ & $6.00 \pm 0.75$ & $6.00 \pm 0.68$ & $6.10 \pm 0.44$ \\
\hline \multirow[t]{2}{*}{$(\mathrm{Sa})$} & 60 & $6.40 \pm 0.25$ & $6.20 \pm 0.51$ & $6.00 \pm 0.38$ & $6.10 \pm 0.62$ & $6.10 \pm 0.65$ & $6.20 \pm 0.67$ \\
\hline & 70 & $6.20 \pm 0.38$ & $6.00 \pm 0.48$ & $5.90 \pm 0.31$ & $6.00 \pm 0.51$ & $5.90 \pm 0.62$ & $6.00 \pm 0.53$ \\
\hline Saag & 70 & $6.20 \pm 0.40$ & $6.10 \pm 0.88$ & $6.00 \pm 0.67$ & $6.50 \pm 0.62$ & $5.70 \pm 0.45$ & $6.10 \pm 0.47$ \\
\hline$(\mathrm{Sa})$ & 80 & $6.30 \pm 0.31$ & $6.20 \pm 0.71$ & $6.10 \pm 0.52$ & $6.60 \pm 0.45$ & $5.80 \pm 0.52$ & $6.20 \pm 0.45$ \\
\hline Pulao & 50 & $6.00 \pm 0.54$ & $5.90 \pm 0.65$ & $6.00 \pm 0.62$ & $5.70 \pm 0.82$ & $5.20 \pm 0.72$ & $5.80 \pm 0.65$ \\
\hline \multirow[t]{2}{*}{$(\mathrm{Sa})$} & 60 & $6.10 \pm 0.42$ & $6.10 \pm 0.78$ & $6.10 \pm 0.55$ & $5.90 \pm 0.71$ & $5.20 \pm 0.65$ & $5.90 \pm 0.71$ \\
\hline & 70 & $5.90 \pm 0.32$ & $5.90 \pm 0.71$ & $6.00 \pm 0.43$ & $5.60 \pm 0.65$ & $5.10 \pm 0.50$ & $5.70 \pm 0.51$ \\
\hline Kadhi & 30 & $6.60 \pm 0.09$ & $6.50 \pm 0.18$ & $6.70 \pm 0.12$ & $6.20 \pm 0.16$ & $6.10 \pm 0.08$ & $6.40 \pm 0.15$ \\
\hline \multirow[t]{2}{*}{$(\mathrm{Sa})$} & 40 & $6.80 \pm 0.02$ & $6.80 \pm 0.13$ & $6.80 \pm 0.05$ & $6.80 \pm 0.12$ & $6.70 \pm 0.14$ & $6.80 \pm 0.21$ \\
\hline & 50 & $6.20 \pm 0.12$ & $6.30 \pm 0.08$ & $6.10 \pm 0.10$ & $6.10 \pm 0.09$ & $6.00 \pm 0.11$ & $6.10 \pm 0.09$ \\
\hline$I d l i$ & 40 & $6.40 \pm 0.52$ & $6.50 \pm 0.89$ & $6.10 \pm 0.34$ & $6.20 \pm 0.53$ & $6.10 \pm 0.43$ & $6.30 \pm 0.39$ \\
\hline \multirow[t]{2}{*}{ (Sa) } & 50 & $6.80 \pm 0.48$ & $6.70 \pm 0.68$ & $6.20 \pm 0.45$ & $6.60 \pm 0.59$ & $6.40 \pm 0.36$ & $6.50 \pm 0.48$ \\
\hline & 60 & $6.10 \pm 0.43$ & $6.10 \pm 0.73$ & $6.00 \pm 0.38$ & $6.10 \pm 0.56$ & $6.00 \pm 0.39$ & $6.10 \pm 0.52$ \\
\hline Soup & 60 & $5.60 \pm 0.51$ & $5.70 \pm 0.21$ & $5.90 \pm 0.29$ & $6.10 \pm 0.41$ & $5.50 \pm 0.45$ & $5.70 \pm 0.29$ \\
\hline \multirow{2}{*}{$(\mathrm{Sa})$} & 70 & $5.90 \pm 0.31$ & $5.80 \pm 0.27$ & $6.00 \pm 0.48$ & $6.20 \pm 0.52$ & $5.80 \pm 0.27$ & $5.99 \pm 0.63$ \\
\hline & 80 & $5.50 \pm 0.45$ & $5.60 \pm 0.18$ & $5.60 \pm 0.31$ & $5.80 \pm 0.38$ & $5.30 \pm 0.19$ & $5.50 \pm 0.41$ \\
\hline Sweet & 60 & $6.00 \pm 0.35$ & $6.00 \pm 0.75$ & $6.00 \pm 0.62$ & $5.70 \pm 0.82$ & $5.20 \pm 0.72$ & $5.80 \pm 0.34$ \\
\hline Pulao & 70 & $6.10 \pm 0.49$ & $6.00 \pm 0.62$ & $6.10 \pm 0.55$ & $5.90 \pm 0.71$ & $5.20 \pm 0.65$ & $5.90 \pm 0.53$ \\
\hline$(\mathrm{Sw})$ & 80 & $5.90 \pm 0.25$ & $5.90 \pm 0.58$ & $6.00 \pm 0.43$ & $5.60 \pm 0.65$ & $5.10 \pm 0.50$ & $5.70 \pm 0.48$ \\
\hline Gulabjamun & 40 & $6.70 \pm 0.41$ & $6.80 \pm 0.40$ & $6.80 \pm 0.87$ & $6.50 \pm 0.81$ & $6.90 \pm 0.56$ & $6.70 \pm 0.38$ \\
\hline \multirow[t]{2}{*}{$(\mathrm{Sw})$} & 50 & $6.90 \pm 0.45$ & $6.90 \pm 0.32$ & $7.00 \pm 0.82$ & $7.00 \pm 0.82$ & $7.00 \pm 0.65$ & $6.96 \pm 0.27$ \\
\hline & 60 & $6.30 \pm 0.34$ & $6.60 \pm 0.34$ & $6.70 \pm 0.79$ & $6.20 \pm 0.79$ & $6.70 \pm 0.74$ & $6.50 \pm 0.49$ \\
\hline Gulgulae & 50 & $5.80 \pm 0.45$ & $5.70 \pm 0.38$ & $5.50 \pm 0.41$ & $5.60 \pm 0.32$ & $5.10 \pm 0.45$ & $5.50 \pm 0.21$ \\
\hline \multirow[t]{2}{*}{$(\mathrm{Sw})$} & 60 & $5.80 \pm 0.36$ & $5.70 \pm 0.35$ & $5.50 \pm 0.39$ & $5.80 \pm 0.28$ & $5.20 \pm 0.42$ & $5.60 \pm 0.32$ \\
\hline & 70 & $5.70 \pm 0.42$ & $5.60 \pm 0.41$ & $5.40 \pm 0.32$ & $5.50 \pm 0.25$ & $5.00 \pm 0.38$ & $5.40 \pm 0.25$ \\
\hline Pancake & 40 & $6.10 \pm 0.31$ & $6.40 \pm 0.35$ & $6.00 \pm 0.35$ & $6.10 \pm 0.67$ & $5.90 \pm 0.68$ & $6.10 \pm 0.32$ \\
\hline \multirow[t]{2}{*}{$(\mathrm{Sw})$} & 50 & $6.20 \pm 0.25$ & $6.50 \pm 0.41$ & $6.10 \pm 0.32$ & $6.20 \pm 0.58$ & $6.00 \pm 0.76$ & $6.20 \pm 0.41$ \\
\hline & 60 & $6.00 \pm 0.29$ & $6.30 \pm 0.32$ & $5.90 \pm 0.28$ & $6.00 \pm 0.65$ & $5.90 \pm 0.81$ & $6.00 \pm 0.27$ \\
\hline & 40 & $6.50 \pm 0.84$ & $6.70 \pm 0.34$ & $6.80 \pm 0.71$ & $6.80 \pm 0.78$ & $6.80 \pm 0.42$ & $6.70 \pm 0.22$ \\
\hline \multirow{2}{*}{ (Sw) } & 50 & $6.80 \pm 0.78$ & $6.90 \pm 0.42$ & $7.00 \pm 0.88$ & $7.00 \pm 0.84$ & $7.00 \pm 0.62$ & $6.90 \pm 0.38$ \\
\hline & 60 & $6.40 \pm 0.58$ & $6.50 \pm 0.38$ & $6.70 \pm 0.75$ & $6.60 \pm 0.69$ & $6.70 \pm 0.74$ & $6.60 \pm 0.42$ \\
\hline
\end{tabular}

Values are Mean \pm S.E.

Sw-Sweet Preparation; Sa-Safty Preparation

and the scores for overall acceptability ranged $5.60 \pm 0.32$ (gulgulae) to $6.40 \pm 0.44$ (chutney). Whereas, sweet pulao and soup were best acceptable at 70 per cent level of incorporation and respective scores for overall acceptability were $5.90 \pm 0.53$ and $5.99 \pm 0.63$. Saag scored highest scores at 80 per cent level of incorporation of fresh greens and the best scores for overall acceptability awarded by the panel of judges was $6.20 \pm 0.45$.

Fresh and Dry Carrot Greens Incorporated Food Preparations: Mathi, matrey, titbits, tacques and laddoo were developed by incorporating both fresh carrot greens as well as dry carrot greens separately at different levels. For mathri, best level of incorporation of fresh carrot greens was found to be 60 per cent and 9 per cent of dry carrot greens and the scores for overall acceptability were $6.09 \pm 0.52$ for fresh greens and $6.05 \pm 0.63$ for dry greens, respectively. Matrey, titbits, tacques and laddoo were best acceptable at 50 per cent level of incorporation of fresh greens and the respective scores for overall acceptability ranged from $5.52 \pm 0.68$ 
Table 3: Sensory evaluation of different food preparations with incorporation of both fresh and dry leaves of underexploited greens of carrot

\begin{tabular}{|c|c|c|c|c|c|c|c|}
\hline $\begin{array}{l}\text { Food } \\
\text { prepara- } \\
\text { tions }\end{array}$ & $\begin{array}{l}\% \text { level } \\
\text { of incor- } \\
\text { poration }\end{array}$ & Appearance & Color & Texture & Taste & Flavour & $\begin{array}{c}\text { Overall } \\
\text { acceptability }\end{array}$ \\
\hline Mathri & 50 & $5.80 \pm 0.45$ & $5.90 \pm 0.41$ & $6.00 \pm 0.99$ & $6.05 \pm 0.28$ & $6.00 \pm 0.83$ & $5.95 \pm 0.32$ \\
\hline \multirow{2}{*}{$(f c g)$} & 60 & $5.90 \pm 0.32$ & $6.10 \pm 0.48$ & $6.10 \pm 0.85$ & $6.10 \pm 0.25$ & $6.10 \pm 0.76$ & $6.09 \pm 0.52$ \\
\hline & 70 & $5.70 \pm 0.42$ & $5.80 \pm 0.30$ & $5.90 \pm 0.92$ & $6.00 \pm 0.21$ & $5.90 \pm 0.69$ & $5.89 \pm 0.61$ \\
\hline Mathri & 8 & $5.90 \pm 0.54$ & $5.90 \pm 0.62$ & $5.90 \pm 0.79$ & $6.00 \pm 0.55$ & $5.90 \pm 0.85$ & $5.93 \pm 0.72$ \\
\hline \multirow[t]{2}{*}{$(\mathrm{dcg})$} & 9 & $6.00 \pm 0.42$ & $6.10 \pm 0.45$ & $6.00 \pm 0.65$ & $6.10 \pm 0.70$ & $6.00 \pm 0.69$ & $6.05 \pm 0.63$ \\
\hline & 10 & $5.90 \pm 0.31$ & $5.80 \pm 0.53$ & $5.80 \pm 0.71$ & $5.90 \pm 0.82$ & $5.70 \pm 0.72$ & $5.86 \pm 0.59$ \\
\hline Matrey & 40 & $5.90 \pm 0.64$ & $5.80 \pm 0.68$ & $6.05 \pm 0.84$ & $6.10 \pm 0.72$ & $5.95 \pm 0.79$ & $5.98 \pm 0.55$ \\
\hline \multirow[t]{2}{*}{$(\mathrm{fcg})$} & 50 & $6.10 \pm 0.62$ & $6.00 \pm 0.74$ & $6.20 \pm 0.78$ & $6.20 \pm 0.78$ & $6.05 \pm 0.81$ & $6.12 \pm 0.72$ \\
\hline & 60 & $6.00 \pm 0.59$ & $5.70 \pm 0.65$ & $6.00 \pm 0.75$ & $6.00 \pm 0.67$ & $5.90 \pm 0.77$ & $5.94 \pm 0.61$ \\
\hline Matrey & 6 & $5.90 \pm 0.55$ & $5.80 \pm 0.74$ & $6.05 \pm 0.84$ & $6.10 \pm 0.72$ & $5.95 \pm 0.81$ & $5.97 \pm 0.58$ \\
\hline \multirow[t]{2}{*}{$(\mathrm{dcg})$} & 7 & $6.00 \pm 0.62$ & $6.00 \pm 0.68$ & $6.20 \pm 0.78$ & $6.20 \pm 0.65$ & $6.05 \pm 0.65$ & $6.11 \pm 0.67$ \\
\hline & 8 & $5.90 \pm 0.45$ & $5.70 \pm 0.65$ & $6.00 \pm 0.75$ & $6.00 \pm 0.78$ & $5.90 \pm 0.77$ & $5.93 \pm 0.74$ \\
\hline Titbits & 40 & $6.20 \pm 0.54$ & $6.10 \pm 0.59$ & $6.00 \pm 0.41$ & $6.00 \pm 1.01$ & $5.90 \pm 0.73$ & $6.04 \pm 0.35$ \\
\hline \multirow[t]{2}{*}{$(f c g)$} & 50 & $6.30 \pm 0.52$ & $6.20 \pm 0.64$ & $6.20 \pm 0.50$ & $6.10 \pm 1.13$ & $6.10 \pm 0.65$ & $6.19 \pm 0.88$ \\
\hline & 60 & $6.00 \pm 0.21$ & $5.90 \pm 0.53$ & $5.70 \pm 0.37$ & $5.80 \pm 0.99$ & $5.80 \pm 0.69$ & $5.85 \pm 0.52$ \\
\hline Titbits & 7 & $6.30 \pm 0.42$ & $6.20 \pm 0.39$ & $6.10 \pm 0.21$ & $6.10 \pm 1.31$ & $6.00 \pm 0.45$ & $6.15 \pm 0.53$ \\
\hline \multirow[t]{2}{*}{ (dcg) } & 8 & $6.40 \pm 0.23$ & $6.30 \pm 0.24$ & $6.10 \pm 0.30$ & $6.10 \pm 1.34$ & $6.15 \pm 0.31$ & $6.24 \pm 0.41$ \\
\hline & 9 & $6.10 \pm 0.28$ & $5.20 \pm 0.43$ & $6.00 \pm 0.34$ & $5.90 \pm 0.29$ & $6.05 \pm 0.48$ & $5.87 \pm 0.33$ \\
\hline Tacques & 40 & $6.20 \pm 0.15$ & $6.20 \pm 0.12$ & $5.90 \pm 0.21$ & $6.50 \pm 0.18$ & $6.10 \pm 0.22$ & $6.19 \pm 0.23$ \\
\hline \multirow[t]{2}{*}{$(\mathrm{fcg})$} & 50 & $6.50 \pm 0.21$ & $6.60 \pm 0.19$ & $6.10 \pm 0.25$ & $6.70 \pm 0.15$ & $6.15 \pm 0.19$ & $6.42 \pm 0.43$ \\
\hline & 60 & $6.10 \pm 0.19$ & $6.20 \pm 0.15$ & $5.70 \pm 0.12$ & $6.40 \pm 0.23$ & $6.00 \pm 0.15$ & $6.11 \pm 0.35$ \\
\hline Tacques & 7 & $6.20 \pm 0.25$ & $6.20 \pm 0.12$ & $5.80 \pm 0.15$ & $6.40 \pm 0.31$ & $6.00 \pm 0.25$ & $6.12 \pm 0.19$ \\
\hline \multirow[t]{2}{*}{$(\mathrm{dcg})$} & 8 & $6.40 \pm 0.31$ & $6.30 \pm 0.15$ & $6.00 \pm 0.21$ & $6.50 \pm 0.35$ & $6.25 \pm 0.20$ & $6.31 \pm 0.28$ \\
\hline & 9 & $6.10 \pm 0.29$ & $6.10 \pm 0.21$ & $5.80 \pm 0.19$ & $6.20 \pm 0.19$ & $6.00 \pm 0.19$ & $6.06 \pm 0.32$ \\
\hline Laddoo & 40 & $5.30 \pm 0.72$ & $5.60 \pm 0.51$ & $5.50 \pm 0.98$ & $5.20 \pm 0.63$ & $5.10 \pm 0.60$ & $5.34 \pm 0.65$ \\
\hline \multirow[t]{2}{*}{$(\mathrm{fcg})$} & 50 & $5.50 \pm 0.90$ & $5.70 \pm 0.64$ & $5.70 \pm 0.74$ & $5.50 \pm 0.56$ & $5.15 \pm 0.59$ & $5.52 \pm 0.68$ \\
\hline & 60 & $5.20 \pm 0.85$ & $5.40 \pm 0.58$ & $5.30 \pm 0.61$ & $4.95 \pm 0.48$ & $4.95 \pm 0.47$ & $5.17 \pm 0.59$ \\
\hline \multirow{3}{*}{$\begin{array}{l}\text { Laddoo } \\
\text { (dcg) }\end{array}$} & 7 & $5.90 \pm 0.51$ & $6.00 \pm 0.72$ & $5.80 \pm 0.58$ & $5.95 \pm 0.51$ & $5.40 \pm 0.58$ & $5.82 \pm 0.63$ \\
\hline & 8 & $6.05 \pm 0.63$ & $6.10 \pm 0.63$ & $5.90 \pm 0.64$ & $6.10 \pm 0.64$ & $5.65 \pm 0.39$ & $5.98 \pm 0.69$ \\
\hline & 9 & $5.80 \pm 0.48$ & $5.90 \pm 0.59$ & $5.60 \pm 0.42$ & $5.70 \pm 0.43$ & $5.15 \pm 0.43$ & $5.66 \pm 0.54$ \\
\hline
\end{tabular}

Values are Mean $\pm \mathrm{SE}$

dcg : preparations prepared by incorporating dry carrot greens.

fcg : preparations prepared by incorporating fresh carrot greens.

(laddoo) to $6.42 \pm 0.43$ (tacques). Titbits, laddoo and tacques were best acceptable at 8 per cent level of incorporation of dry carrot greens and scores for overall acceptability ranged from 5.98 \pm 0.69 (laddoo) to $6.31 \pm 0.28$ (tacques). Matrey were found to be most acceptable at 7 per cent level of dry carrot greens incorporation and the best scores for overall acceptability awarded by the panel of judges was $6.11 \pm 0.67$. Statistical analysis for overall acceptability among all food preparations showed that judges were highly consistent in awarding the scores for gulabjamun (6.96), rabri (6.90), kadhi (6.80), idli (6.50), chutney (6.40), tacques (6.42), titbits (6.24), cutlets, saag, puri, pancake and chilla (6.20), matrey (6.12) and mathri (6.09).

$\beta$ Carotene Content: In all controlled (without incorporation) food preparations, $\beta$ carotene content was zero barring soup, saag and chutney but when these food preparations were prepared with incorporation of under exploited greens of carrot either in dry (mathri, matrey, tacques, titbits) or fresh form (puri, kadhi, pancake, rabri, idli, laddoo, gulabjamun, chilla, pulao, guglae, sweet pulao, soup, saag, mathri, matrey, tacques and titbits) at different levels (7, 8, 9, 40, 50, 60, 70 and $80 \%$ ) their â carotene content significantly $(\mathrm{P} \leq 0.01)$ increased from 2280 to $4560 \mu \mathrm{g} / 100 \mathrm{~g}$. The daily requirement of $\beta$ carotene of a child / an adult person / ranges from $1200 \mu \mathrm{g}$ to $2400 \mu \mathrm{g}$ as recommended by ICMR (1989) and this can be fulfilled by one serving of any of the above said preparations.

\section{CONCLUSION AND RECOMMENDATIONS}

Conclusion: All the food products developed with incorporation of greens either in dry form or 
Table 4: $\beta$ carotene content of the most acceptable food preparations based on underexploited carrot greens (UCG)

\begin{tabular}{|c|c|c|c|c|c|}
\hline \multirow[t]{3}{*}{ Food preparations } & \multicolumn{2}{|c|}{ Per cent level of incorporation of $U C G$} & \multicolumn{3}{|c|}{$\beta$ carotene $(u g / 100 g) \$$} \\
\hline & \multirow[b]{2}{*}{ Fresh } & \multirow[b]{2}{*}{ Dry } & \multirow{2}{*}{$\begin{array}{c}\text { Without } \\
\text { incorporation } \\
\text { of UCG }\end{array}$} & \multicolumn{2}{|c|}{$\begin{array}{c}\text { With incorporation } \\
\text { of UCG }\end{array}$} \\
\hline & & & & Fresh\# & Dry\# \\
\hline Puri & 40 & - & - & 2280 & - \\
\hline Kadhi & 40 & - & - & 2280 & - \\
\hline Matrey & 50 & - & - & 2850 & - \\
\hline Matrey & - & 7 & - & - & 2280 \\
\hline Tacques & 50 & - & - & 2850 & - \\
\hline Tacques & - & 8 & - & - & 2850 \\
\hline Titbits & 50 & - & - & 2850 & - \\
\hline Titbits & - & 8 & - & - & 2850 \\
\hline Pancake & 50 & - & - & 2850 & - \\
\hline Rabri & 50 & - & - & 2850 & - \\
\hline Idli & 50 & - & - & 2850 & - \\
\hline Laddoo & 50 & - & - & 2850 & - \\
\hline Laddoo & - & 8 & - & - & 2850 \\
\hline Gulabjamun & 50 & - & - & 2850 & - \\
\hline Chilla & 60 & - & - & 3420 & - \\
\hline Pulao & 60 & - & - & 3420 & - \\
\hline Mathri & 60 & - & - & 3420 & - \\
\hline Mathri & - & 9 & - & - & 3420 \\
\hline Gulgulae & 60 & - & - & 3420 & - \\
\hline Cutlet & 60 & - & - & 3420 & - \\
\hline Chutney & 60 & - & *3091 & 3420 & - \\
\hline Sweet pulao & 70 & - & - & 3990 & - \\
\hline Soup & 70 & - & $* * 4006$ & 3990 & - \\
\hline Saag & 80 & - & $* * * 2097$ & 4560 & - \\
\hline
\end{tabular}

* coriander/Mint(2:1); ** Spinach; ***Mustard

$\$$ Gopalan C. (1989)

\# significant at $\mathrm{P} \leq 0.01$

fresh form were organoleptically acceptable. $\beta$ carotene content in all food preparations increased significantly $(\mathrm{P} \leq 0.01)$ with incorporation of fresh as well as dry carrot greens. Analysis of data further showed that increase of $\beta$ carotene was more in food preparations which were prepared with incorporation of fresh carrot greens compared to dry carrot greens.

Recommendations: It is strongly recommended that most of the commonly consumed food preparations can be successfully prepared by incorporating under exploited carrot greens and these preparations if consumed on a daily basis can take care of the micronutrient deficiencies of the population.

\section{REFERENCES}

Gopalan C 1992. New dimensions of old problem. In: Nutrition in development transition in South-East Asia. WHO. Regional office for South-East Asia. New Delhi, pp. 34-38.

Gopalan C, Ramasastri BV, Balasubramanian SC, Narasinga Rao BS, Deosthale YG, Pant KC 2004. Nutritive value of Indian foods: Indian Council of Medical Research, Hyderabad: National Institute of Nutrition.

Gopalan C 1996. An overview-combating vitamin A deficiency and micronutrient malnutrition through dietary improvement. In: Subhadra Seshadri (Eds.): Use of Carotene Rich Foods to Combat Vitamin A Deficiency in India - A Multycentric Study. New Delhi: Nutrition Foundation of India.

Gupta JP, Saini SS 1987. Introduction to Statistical Methods. New Delhi: Kalyani Publishers.

Hopkins JW 1950. A procedure for quantifying subjective appraisals of odor, flavor, and texture of foodstuffs. Biometrics, 6(1):1.

ICMR 1989. Nutritive Value of Indian Foods. Hyderabad: NIN, ICMR.

Kaur Tarvinder Jeet 2007. Development of Low-cost Nutritious Recipes Using Discarded Greens. A Report (unpublished) submitted to Department of Home Science, Kurukshetra University, Kurukshetra, India.

National Programme for Control of Blindness 1981. Report of National Workshop. New Delhi: Directorate General of Health Services, India.

Negi Pradeep Singh, Roy Susanta Kumar 2004. Changes in beta carotene and ascorbic acid content of fresh amaranth and fenugreek leaves during storage by low cost technique. Plant Foods Hum Nutr, 58: 225-230. 
Ramadasmurthy V, Mohanram M 1984. Nutritional blindness due to vitamin A deficiency.Your Health and Nutrition. Hyderabad: NIN.

Thylefors B 1985. Prevention of blindness the current focus. WHO Chronicle, 39(4): 150.
UNDP, Human Development Report 1992. FAO/WHO, Report on the World Nutrition Challenge; UNICEF, Care and Child Nutrition, ACC/SCN, Report on the World Nutrition Situation and Update. WHO, Bulletin 70(2); World Health Statistics Quarterly, 38 (3). 